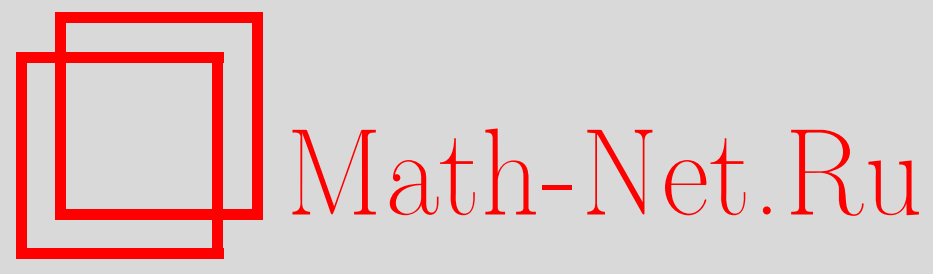

Г. Т. Булгакова, Н. Р. Кондратьева, Аналитическая модель вертикального вытеснения нефти водой с учётом вязкостных, гравитационных и капиллярных сил, Вестн. Сам. гос. техн. ун-та. Сер. Физ.-мат. науки, 2012, выпуск 1(), 208-213

DOI: https://doi.org/10.14498/vsgtu1006

Использование Общероссийского математического портала Math-Net.Ru подразумевает, что вы прочитали и согласны с пользовательским соглашением

http: //www . mathnet.ru/rus/agreement

Параметры загрузки:

IP: 18.209 .158 .208

26 апреля 2023 г., 17:23:11

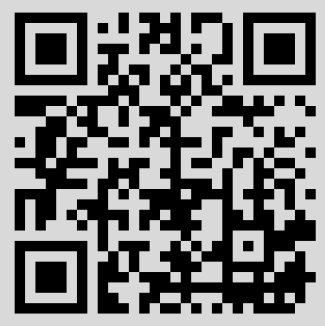


УДК 517.958:532.5.013

\title{
АНАЛИТИЧЕСКАЯ МОДЕЛЬ ВЕРТИКАЛЬНОГО ВЫТЕСНЕНИЯ НЕФТИ ВОДОЙ С УЧЕТОМ ВЯЗКОСТНЫХ, ГРАВИТАЦИОННЫХ И КАПИЛЛЯРНЫХ СИЛ
}

\section{Г. Т. Булгакова, Н.Р. Кондратъева}

Уфимский государственный технический университет, 450000, Россия, Уфа, ул. К. Маркса, 12.

E-mail: kondratyevanr@mail.ru

\begin{abstract}
Представлена аналитическая модель одномерного водонефтяного вытеснения, которая учитывает три основные силы: капиллярные, гравитационнье и вязкостные. Данная математическая модель имеет вид нелинейного параболического дифференииального уравнения второго порядка. В симуляторе Schlumberger ECLIPSE ver. 2004a_1 построена гидродинамическая модель рассматриваемого процесса. Результат̆ы численного моделирования сравниваются с аналитическим решением задачи.
\end{abstract}

Ключевые слова: гидродинамическое моделирование, математическое моделирование, капиллярные силь.

Введение. Широкое распространение методов заводнения сделало вытеснение нефти водой из пластов основным процессом разработки месторождений. Как и любой другой, метод заводнения нуждается в предварительном изучении для выбора лучших условий добычи. Анализ одномерных течений позволяет выявить основные эффекты и характерные особенности фильтрации жидкостей.

Эта работа представляет аналитическую модель для одномерного водонефтяного вытеснения, которая учитывает три основные силы: капиллярные, гравитационные и вязкостные.

Модель в безразмерных переменных принимает вид нелинейного параболического дифференциального уравнения второго порядка, которое предполагает, что каждая из трех сил включается независимо. Влияние вязкости воды, скорости закачиваемого потока, величины капиллярного давления рассмотрено в структуре безразмерного гравитационного числа и безразмерного капиллярного числа. Аналитическое решение полученного дифференциального уравнения сопоставляется с результатами гидродинамического моделирования.

Модель вытеснения. Математическая модель потока двух несмешивающихся флюидов в однородном линейном резервуаре базируется на двух основных уравнениях: уравнение сохранения массы и уравнение скорости [1]. Скорость каждой фазы описывается законом Дарси:

$$
u_{w}=-k \lambda_{r w}\left(S_{w}\right)\left[\frac{\partial p_{w}}{\partial z}+\gamma_{w} \frac{\partial H}{\partial z}\right], \quad u_{o}=-k \lambda_{r o}\left(S_{w}\right)\left[\frac{\partial p_{o}}{\partial z}+\gamma_{o} \frac{\partial H}{\partial z}\right] .
$$

Здесь $u_{i}$ - скорость $i$-той фазы, $k$ - абсолютная проницаемость, $\lambda_{r i}\left(S_{i}\right)$ - относительная подвижность $i$-той фазы, $p_{i}$ - давление $i$-той фазы, $H$ - глубина,

Гузель Талгатовна Булгакова (д.ф.-м.н., проф.), профессор, каф. математики. Найля Рашитовна Кондратъева, аспирант, каф. математики. 
$z$ - координата, $\gamma_{i}=g \rho_{i}, g$ - ускорение свободного падения, $\rho_{i}-$ плотность $i$ той фазы. Насыщенности фаз связаны следующим уравнением:

$$
S_{w}+S_{o}=1
$$

Капиллярное давление определяется как

$$
P_{c}\left(S_{w}\right)=P_{o}-P_{w} .
$$

При условии несжимаемости флюидов и неизменной общей добычи через единицу площади сечения следует, что

$$
u_{t}=u_{w}+u_{o}
$$

Уравнение сохранения массы имеет вид

$$
\varphi \frac{\partial S}{\partial t}+\frac{\partial u_{w}}{\partial z}=0
$$

где $\varphi-$ пористость.

При комбинировании уравнений (1)-(5) скорость воды может быть описана как

$$
u_{w}=k^{*} \lambda_{r o} \frac{\lambda_{r w}}{\lambda_{r t}}\left[\frac{d p_{c}}{d S_{w}} \frac{\partial S_{w}}{\partial z}-\Delta \gamma \frac{\partial H}{\partial z}\right]+\frac{\lambda_{r w}}{\lambda_{r t}} u_{t}
$$

где $\lambda_{r t}=\lambda_{r w}+\lambda_{r o}-$ общая подвижность. Подставляя (6) в $(5)$, получим

$$
\varphi \frac{\partial S_{w}}{\partial t}+\frac{\partial}{\partial z}\left(k \lambda_{r o} \frac{\lambda_{r w}}{\lambda_{r t}} \frac{d p_{c}}{d S_{w}} \frac{\partial S_{w}}{\partial z}-k \lambda_{r o} \frac{\lambda_{r w}}{\lambda_{r t}} \Delta \gamma \frac{\partial H}{\partial z}+\frac{\lambda_{r w}}{\lambda_{r t}} u_{t}\right)=0 .
$$

Первое слагаемое уравнения (7) определяет вклад капиллярных сил, второе вклад гравитационных сил и третье слагаемое характеризует силы вязкого трения.

Приведём уравнение (7) к безразмерному виду по аналогии с принципами, изложенными в [2, с. 181-276]. Введём следующие безразмерные переменные:

$$
\begin{gathered}
S=\frac{S_{w}-S_{i w}}{1-S_{o r}-S_{i w}}, \quad \varphi^{*}=\varphi\left(1-S_{o r}-S_{i w}\right), \\
z_{D}=z / L, \quad H_{D}=H / L, \quad t_{D}=u_{t} / \varphi^{*} L t .
\end{gathered}
$$

Здесь $S_{i w}, S_{o r}$ - насыщенности связной водой и остаточной нефтью соответственно. Представим функции относительных фазовых проницаемостей и капиллярного давления как функции от насыщенности:

$$
\begin{gathered}
\lambda_{r w}=\lambda_{r w}^{*}(S)^{n w}=\frac{k_{r w}^{*}}{\mu_{w}}(S)^{n w} \\
\lambda_{r o}=\lambda_{r o}^{*}(1-S)^{n o}=\frac{k_{r o}^{*}}{\mu_{o}}(1-S)^{n o}, \quad p_{c}=-p_{c}^{*} \ln (S),
\end{gathered}
$$

где $k_{r w}^{*}, k_{r o}^{*}$ - предельные точки кривых относительной фазовой проницаемости для воды и нефти соответственно, $p_{c}^{*}$ - параметр функции капиллярного давления. В итоге получим

$$
\frac{\partial}{\partial z_{D}}\left[\frac{1}{N_{P e}} D_{C}(S) \frac{\partial S}{\partial z_{D}}+N_{G} G(S)-V(S)\right]-\frac{\partial S}{\partial t_{D}}=0 .
$$


Здесь

$$
N_{P_{e}}=\frac{U_{t} L}{k \lambda_{r w}^{*} p_{c}^{*}}
$$

- безразмерное капиллярное число;

$$
N_{G}=\frac{k \lambda_{r w}^{*} \Delta \gamma}{u_{t}} \frac{\partial H_{D}}{\partial z_{D}}
$$

- безразмерное гравитационное число;

$$
\begin{gathered}
D_{C}(S)=\frac{(1-S)^{n o} S^{n w-1}}{(1-S)^{n o}+M S^{n w}}, \quad G(S)=\frac{(1-S)^{n o} S^{n w}}{(1-S)^{n o}+M S^{n w}} \\
V(S)=\frac{M_{S^{n w}}}{(1-S)^{n o}+M S^{n w}}, \quad M=\frac{\lambda_{r w}^{*}}{\lambda_{r o}^{*}}
\end{gathered}
$$

Аналитическое решение. Уравнение (8) дополним начальным и граничным условиями:

$$
S\left(z_{D}, t_{D}=0\right)=0, \quad S\left(z_{D}=0, t_{D}\right)=1 .
$$

Важно наиболее полно оценить влияние капиллярных и гравитационных сил на процесс вытеснения, поэтому задача о вертикальном вытеснении нефти водой решается для произвольных значений гравитационных и капиллярных сил. Решение ищется для случая $N_{P e}=m, D_{C}=1, V=S, G=S, N_{G}=n$, где $m \neq 0$ и $n \neq 1$ - произвольные числа.

Тогда (8) примет вид

$$
\frac{\partial S}{\partial t_{D}}=\frac{1}{m} \frac{\partial^{2} S}{\partial z_{D}^{2}}+(n-1) \frac{\partial S}{\partial z_{D}}
$$

После приведения уравнения (11) к каноническому виду получаем задачу для нахождения функции $V\left(z_{D}, t_{D}\right)$, которая связана с функцией $S\left(z_{D}, t_{D}\right)$ :

$$
S\left(z_{D}, t_{D}\right)=\exp \left(-\frac{(n-1) m}{2} z_{D}-\frac{(n-1)^{2} m}{4} t_{D}\right) V\left(z_{D}, t_{D}\right)
$$

Для нахождения функции $V\left(z_{D}, t_{D}\right)$ решается следующая краевая задача [3, 242-249]:

$$
V_{t_{D}}^{\prime}=\frac{1}{m} V_{z_{D} z_{D}}^{\prime \prime} ; \quad V\left(z_{D}, t_{D}=0\right)=0, \quad V\left(z_{D}=0, t_{D}\right)=\exp \left(\frac{(n-1)^{2} m}{4} t_{D}\right) .
$$

Отсюда

$$
V\left(z_{D}, t_{D}\right)=\exp \left(\frac{(n-1)^{2} m}{4} t_{D}\right) \operatorname{erfc}\left(\frac{z_{D} \sqrt{m}}{2 \sqrt{t_{D}}}\right),
$$

и решение задачи (11), (10) представляется как

$$
S\left(z_{D}, t_{D}\right)=\exp \left(\frac{(n-1)_{m}}{2} z_{D}\right) \operatorname{erfc}\left(\frac{z_{D} \sqrt{m}}{2 \sqrt{t_{D}}}\right) .
$$


На рис. 1 представлено изменение во времени профиля водонасыщенности, полученного из решения задачи (11), (10).

Оценим характер изменения профиля водонасыщенности в зависимости от значений гравитационного числа $N_{G}$ и капиллярного числа $N_{P_{e}}$. Рассмотрим несколько вариантов: 1) $\left.N_{G}=0, N_{P_{e}}=0,5 ; 2\right) N_{G}=0,196, N_{P_{e}}=0,5$ (направление гравитационного числа и направление закачки совпадают, т. е. $\left.\partial H_{D} / \partial z_{D}>0\right)$; 3) $N_{G}=-0,196, N_{P_{e}}=0,5$ (направление гравитационного числа и направление закачки различны, т. е. $\left.\partial H_{D} / \partial z_{D}<0\right)$; 4) $N_{G}=0,196$, $N_{P_{e}}=1$ (направление гравитационного числа и направление закачки совпадают, т. е. $\left.\partial H_{D} / \partial z_{D}>0\right)$. Расчёт производился по следующим данным: $u_{t}=0,00001 \mathrm{~m} / \mathrm{c}, k=1 \mathrm{mKм}^{2}, \Delta \gamma=200 \Pi \mathrm{a} / \mathrm{m}_{r w}^{*}=1000(\Pi \mathrm{a} \cdot \mathrm{c})^{-1}, L=50 \mathrm{~m}$, $p_{c}^{*}=10^{6}$ Па; На рис. 2 представлены графики распределения водонасыщенности, соответствующие вариантам, описанным выше.

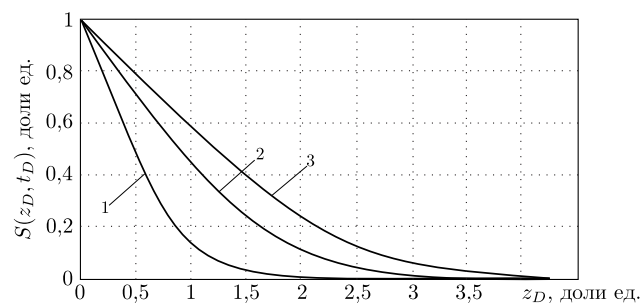

Рис. 1. Профиль водонасыщенности для случая $N_{G}=0,196, N_{P_{e}}=0,5$ в различные моменты времени: 1) $\left.t_{D}=0,1 ; 2\right) t_{D}=0,3$; 3) $t_{D}=0,5$

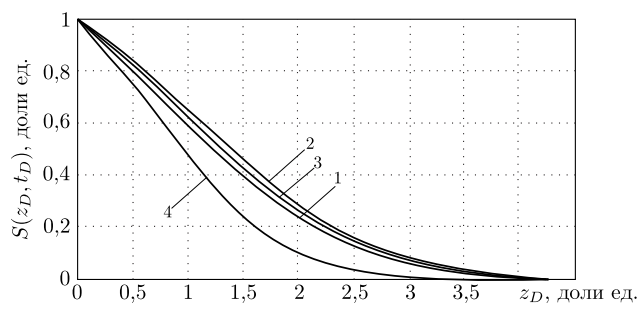

Рис. 2. Профиль водонасыщенности при различных $N_{G}$ и $\left.N_{P_{e}}: 1\right) N_{G}=0,196, N_{P_{e}}=$ $=0,5$;2) $\left.N_{G}=-0,196, N_{P_{e}}=0,5 ; 3\right) N_{G}=0$, $N_{P_{e}}=0,5$; 4) $N_{G}=0,196, N_{P_{e}}=1$

Проведенный анализ чувствительности позволяет оценить вклад гравитационных и капиллярных сил в процесс вытеснения. С ростом числа $N_{P_{e}}$ водонефтяной фронт движется быстрее. Это объясняется тем, что по определению капиллярного числа (9), параметр $N_{P_{e}}$ и капиллярное давление обратно пропорциональны. При больших значениях $N_{P_{e}}$ величина капиллярного давления мала, при этом предполагается, что порода одинаково смачивается и нефтью, и водой. В этом случае капиллярные силы будут противодействовать процессу вытеснения. Представления различных исследователей о механизме проявления и роли капиллярных процессов при вытеснении нефти водой из пористых сред широко описаны в [4]. Влияние гравитации взаимосвязано с направлением потока закачиваемой воды. Если их направления совпадают, то $\partial H_{D} / \partial z_{D}>0$, гравитационные силы будут способствовать процессу вытеснения, и оно будет более эффективным, чем в случае, когда их направления различны.

Численное решение. В гидродинамическом симуляторе Schlumberger ECLIPSE ver.2004a_1 была построена аналогичная модель вытеснения нефти водой для одномерного случая. При расчёте используются следующие свойства флюидов и пористой среды:

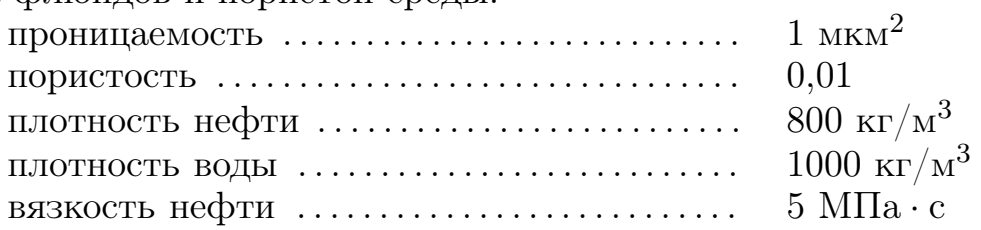


вязкость воды
$1 \mathrm{M \Pi а} \cdot \mathrm{c}$

$5 \cdot 10^{6} ; 10^{6}$ Па

Гидродинамическая модель строилась для двух случаев: 1) $N_{G}=-0,196$, $N_{P_{e}}=0,5$ (направление гравитационного числа и направление закачки различны, т. е. $\left.\partial H_{D} / \partial z_{D}<0\right)$ при $p_{c}^{*}=0,5 \cdot 10^{6}$ Па; 2) $N_{G}=0,196, N_{P_{e}}=1$ (направление гравитационного числа и направление закачки различны, т.е. $\left.\partial H_{D} / \partial z_{D}>0\right)$ при $p_{c}^{*}=10^{6}$ Па. Расчёт производился по следующим данным: $u_{t}=0,00001 \mathrm{~m} / \mathrm{c}, k=1 \mathrm{mKм}^{2}, \Delta \gamma=200 \Pi \mathrm{a} / \mathrm{m}, \lambda_{r w}^{*}=1000(\Pi \mathrm{a} \cdot \mathrm{c})^{-1}$, $L=50$ м. На рис. 3 представлено сравнение профиля водонасыщенности для задачи, решённой аналитически и численно в момент времени $t_{D}=0,5$.

В результате можно говорить о

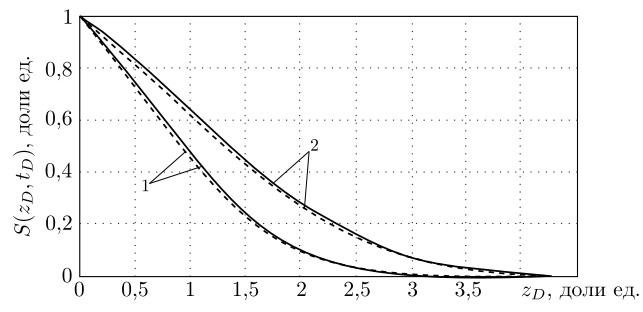

Рис. 3. Сравнение аналитического и численного решения задачи вытеснения: 1) $N_{G}=-0,196$, $\left.N_{P_{e}}=0,5 ; 2\right) N_{G}=0,196, N_{P_{e}}=1$; штриховая линия - численное решение том, что численное решение обеспечивает удовлетворительную сходимость во всей рассматриваемой области. Для выполнения начальных и граничных условий поставленной задачи при заданных числовых параметрах пласта и флюидов в гидродинамической модели число слоев по вертикали следует задавать не менее 300 при толщине слоя 1 м. При увеличении толщины слоя, а значит, и уменьшении общего числа слоёв решить задачу численно невозможно, так как система нелинейных уравнений не имеет решения. Вместе с тем дальнейшее увеличение числа слоев более 300 позволит получить численное решение задачи для последующих моментов времени. Нельзя не отметить, что гидродинамические модели с малым количеством слоев не позволяют в полной мере учитывать все физические процессы, протекающие в пласте.

\section{Выводы.}

1. Представлено уравнение для описания двухфазного несмешивающегося потока в однородной линейной пористой среде. Математическая модель имеет вид нелинейного параболического дифференциального уравнения второго порядка.

2. Получено аналитическое решение задачи о вертикальном вытеснении нефти водой для произвольных значений гравитационных и капиллярных сил в одномерном случае.

3. Определено безразмерное гравитационное число $N_{G}$ и безразмерное капиллярное число $N_{P_{e}}$. Это позволяет оценить влияние каждой силы на процесс вытеснения.

4. В гидродинамическом симуляторе Schlumberger ECLIPSE ver.2004a_1 построена модель рассматриваемого процесса. Результаты численного моделирования сравниваются с аналитическим решением задачи.

\section{БИБЛИОГРАФИЧЕСКИЙ СПИСОК}

1. Aziz Kh., Settari A. Petroleum Reservoir Simulation. London: Applied Science Publishers LTD, 1979. 497 pp.; русск. пер.: Азиз X., Сеттари Э. Математическое моделирование пластовых систем. М.: Наука, 1982. 386 с. 
2. Баренблатт Г.И., Ентов В.М., Рыжик В.М. Теория нестационарной фильтрации жидкости и газа. М.: Недра, 1972. 288 с. [Barenblatt G. I., Yentov V. M., Ryzhik V.M. The theory of the unsteady filtration of liquid and gas. Moscow: Nedra, 1972. 288 pp.]

3. Тихонов А.Н.,Самарский А.А. Уравнения математической физики. М.: Наука, 1977. 741 c. [Tikhonov A. N., Samarskiy A. A. Equations of mathematical physics. Moscow: Nauska, 1977. $741 \mathrm{pp}$.

4. Гиматудинов Ш.К. Физика нефтяного и газового пласта. М.: Недра, 1971. 312 с. [Gimatudinov Sh. K. Physics of the oil and gas reservoir. Moscow: Nedra, 1971. 312 pp.]

Поступила в редакцию $16 / \mathrm{X} / 2011$;

в окончательном варианте - 20/XII/2011.

MSC: 76S05

\section{ANALYTICAL MODEL OF VERTICAL OIL-WATER DISPLACEMENT WITH THE ACCOUNT OF VISCOUS, CAPILLARY AND GRAVITY FORCES}

\section{G. T. Bulgakova, N.R. Kondratyeva}

Ufa State Technical University, 12, K. Marx st., Ufa, 450000, Russia

E-mail: kondratyevanr@mail.ru

A mathematical model of water displacement in vertical porous media is presented. The mathematical formulation takes the form of a nonlinear convection-diffusion equation. Its contribution comes from consideration of the three chief forces: viscous, capillary and gravity in oil recovery processes. A numerical model was based on the simulator Schlumberger ECLIPSE ver. 2004a_1. Finally, analytical and numerical results are compared.

Key words: hydrodynamic modeling, mathematical modeling, gravity forces, capillary forces.

Original article submitted $16 / \mathrm{X} / 2011$ revision submitted 20/XII/2011.

Guzel T. Bulgakova (Ph. D. (Phys. \& Math.)), Professor, Dept. of Mathematics. Nailya R. Kondratyeva, Postgraduate Student, Dept. of Mathematics. 